\title{
Sujeito nulo no português brasileiro: variação estável ou mudança em curso?
}

\section{Null subject in Brazilian Portuguese: stable variation or change in progress?}

\author{
Aline Miyuke Miyamoto* \\ Universidade Estadual de Londrina \\ Londrina, Paraná, Brasil \\ Dircel Aparecida Kailer** \\ Universidade Estadual de Londrina \\ Londrina, Paraná, Brasil
}

Resumo: De acordo com a teoria gerativa, em sua formulação, o parâmetro do sujeito nulo apresentava um valor binário, representado pelos valores negativo ou positivo. Se é uma língua de sujeito nulo, possui um valor positivo (por exemplo, a língua italiana). No entanto, se é uma língua de sujeito preenchido, possui um valor negativo (um exemplo é a língua inglesa). O português brasileiro (PB) é uma língua natural, isto é, transformase com o decorrer do tempo. Assim como outras línguas, o PB apresenta uma categoria diferente, com sujeito nulo "parcial" (DUARTE, 2018). Estudos indicam que há uma predisposição a preencher a posição de sujeito na língua pelos falantes, antes considerada língua de sujeito nulo. Alguns dos fatores são: 1) houve uma reconfiguração no quadro dos pronomes pessoais do caso reto (as formas gramaticalizadas você e a gente são empregadas como pronomes pessoais); 2) a desinência flexional verbal não é mais suficiente para designar a pessoa gramatical/discursiva (eu/você/ele ou ela/a gente falava; nós falávamos; vocês/eles ou elas falavam). Por essas razões, está ocorrendo uma mudança na marcação desse parâmetro. Com base nos dados do Atlas Linguístico do Brasil, pretende-se, como objetivo geral, verificar se o preenchimento com o pronome-sujeito é predominante ou não na fala de 31 informantes desse projeto, distribuídos pelo Brasil em duas regiões e quatro capitais: na Região Sul - Florianópolis e Curitiba e na Região Centro-Oeste - Campo Grande e Cuiabá. Analisase a fala desses 31 informantes e codificam-se os dados por meio do Programa GoldVarb X; consoante às variáveis linguísticas (pessoa gramatical/desinência verbal explícita ou implícita) e às variáveis extralinguísticas (sexo, grau de escolaridade e localidade). Esta pesquisa está alicerçada nos pressupostos teórico-metodológicos da Sociolinguística Quantitativa (LABOV, 2008 [1972]) e da Teoria de Princípios e Parâmetros (CHOMSKY, 1981, 1995). O uso do sujeito pleno foi predominante em ambos os sexos, graus de escolaridades e nas quatro localidades.

Palavras-chave: Atlas linguístico do Brasil. Teoria da variação e mudança linguística. Teoria de princípios e parâmetros. Parâmetro do sujeito nulo.

\begin{abstract}
According to the generative theory, in its formulation, the null subject parameter had a binary value, represented by the negative or positive values. If it is a null subject language, it has a positive value (for example, the Italian language). However, if it is an overt subject language, it has a negative value (an example is the English language). Brazilian Portuguese (PB) is a natural language, that is, it transforms over time, like other languages, the PB presents a different category, with a "partial" null subject (DUARTE, 2018). Studies indicate
\end{abstract}

\footnotetext{
${ }^{*}$ Mestranda do Programa de Pós-Graduação em Letras - PPGL - Universidade Estadual de Londrina, Londrina, Paraná, Brasil. E-mail: alinemiyuke@gmail.com.

**Professora do Programa de Pós-Graduação em Letras - PPGL - Universidade Estadual de Londrina, Londrina, Paraná, Brasil. E-mail: ueldircel@hotmail.com.
} 
that there is a predisposition to express the position of subject in BP by the speakers, previously considered a null subject language. Some of the factors are: 1) there was a reconfiguration in the framework of the personal pronouns (the grammaticalized forms você and a gente are employed as personal pronouns); 2) the verbal inflectional is no longer enough to designate the grammatical / discursive person (I / you / he / she / it / we / we / we / we / they / they). For these reasons, a change in the marking of this parameter is occurring. Based on data of Linguistic Atlas of Brazil; it is intended, as a general objective, to verify if the completion with the pronoun-subject is predominant or not in the speech of 31 informants of this project, distributed in Brazil in two regions and four capitals: in the South Region - Florianópolis and Curitiba and in the Central-West Region - Campo Grande and Cuiabá. The speech of these 31 informants is analyzed and the data is coded through the GoldVarb X Program; according to the linguistic variables (grammatical person / explicit or implicit verbal ending) and to the extralinguistic variables (sex, educational level and locality). This research is based on the theoretical-methodological assumptions of Quantitative Sociolinguistics (LABOV, 2008 [1972]) and on the Theory of Principles and Parameters (CHOMSKY, 1981, 1995). The use of the overt subject was predominant in both sexes, educational levels and in the four localities.

Keywords: Linguistic Atlas of Brazil. Theory of variation and linguistic change. Theory of principles and parameters. Parameter of the null subject.

\section{INTRODUÇÃO}

O PB apresenta um caso diferente de língua de sujeito nulo. Conforme diversos estudos realizados (BOTASSINI, 1998; DUARTE, 1995, 1996 [1993], 2015, 2016, 2018; GALVES, 1996 [1993], 2001; GRAVINA, 2008; MAGALHÃES, 2006; MARINS, 2009; MENON, 1995, 2002; MONTEIRO, 1994; SILVA, 2006, 2011, entre outros), não se comporta como outras línguas românicas, prototípicas de sujeito nulo, como, por exemplo, a língua italiana, o espanhol peninsular e o português europeu, do qual advém; mas também não é uma língua de sujeito preenchido, como o francês. Muito se discutiu sobre as propriedades que caracterizam uma língua de sujeito nulo. Uma abordagem foi o fato de apresentaram paradigma flexional abundante; assim, não havia necessidade de explicitar o sujeito, pois a desinência verbal seria suficiente. Houve a redução desse paradigma flexional no PB: de seis formas, reduziu-se a três (CASTILHO, 2010), com a gramaticalização das formas você, a gente, a perda da concordância de tu e de eles, a flexão de terceira pessoa do singular iguala-se a essas, vocês e eles com concordância também usam a mesma flexão e a primeira pessoa do singular manteve a sua.

Nesta pesquisa, há uma amostra de ocorrências de sujeitos plenos e nulos presentes nas falas de informantes do Atlas Linguístico do Brasil (ALiB). A seguir, encontram-se a apresentação dos pressupostos teóricos que alicerçam o presente estudo, a Teoria da Variação e Mudança Linguística (WEINREICH; LABOV; HERZOG, 2006 [1968]) e a Teoria de Princípios e Parâmetros (CHOMSKY, 1981, 1995), dos pressupostos metodológicos usados para a análise, do desenvolvimento e de algumas considerações finais.

\section{PRESSUPOSTOS TEÓRICOS}

O modelo gerativista nasceu para opor-se ao behaviorismo, em que se pregava o condicionamento social da aquisição da linguagem, ou seja, a criança aprende por 
exposição ao meio em que vive. Sendo assim, na década de 50, o linguista norte-americano Noam Chomsky formulou a Gramática Gerativa, considerando que todo indivíduo possui, em sua genética, uma predisposição a adquirir a linguagem, uma capacidade inata, presente na Gramática Universal (GU) de cada pessoa. Para o estudioso, a língua tem um conjunto finito de elementos, a partir dos quais se consegue formar um número infinito de sentenças.

Além disso, Chomsky (1965) estabeleceu conceitos imprescindíveis, como competência (Língua-I) e desempenho (Língua-E). O conjunto de regras que o falante possui internalizado, o conhecimento que ele tem de sua língua, é denominado competência, já "o uso que fazemos da língua [...] se denomina desempenho. O desempenho é, afinal, aquilo que efetivamente realizamos quando falamos (ou quando ouvimos, ou escrevemos, ou lemos)" (PERINI, 1979, p. 27).

Outra contribuição importante do linguista foi a Teoria de Princípios e Parâmetros, presente na obra Lectures on government and binding, de 1981, sendo os princípios as regras gerais que estão contidas na mente do falante, comum a todas as línguas, e os parâmetros são as divergências encontradas nelas. E um parâmetro que recebeu muita atenção foi o Parâmetro do Sujeito Nulo. Conforme Duarte (1995, p. 2), "não só é o mais popular como um dos que mais têm recebido contribuições e, consequentemente, passado por reformulações ou refinamentos, desde sua apresentação oficial”. Em sua criação, acreditava-se que o sujeito não era foneticamente realizado por causa de uma morfologia rica da língua, pois as desinências verbais eram capazes de expor a pessoa gramatical, no entanto isso foi desmistificado com o trabalho de Huang (1984), que mostrou a ocorrência de sujeitos nulos no chinês, língua em que não há flexão verbal. Com isso, estabeleceu-se que existem níveis de parâmetros, não apenas "valores binários, que podemos representar, por exemplo, pelos valores positivo ou negativo" (MIOTO; SILVA; LOPES, 2016 [1999], p. 32). O PB moderno se encaixa na categoria de língua de sujeito nulo "parcial", em que ainda há o uso do nulo, mas não é mais predominante na língua, como pode ser visto em Duarte (1996 [1993]): em 1845, a ocorrência de sujeitos nulos é 80\%, já em 1882, 70\%, em 1918 é $75 \%$, caindo significativamente em 1937, com 55\%, em 1955, apresentava 50\% de sujeitos nulos, em 1975, um percentual de 32\%, e 25\% em 1992.

A gramática gerativa serve como suporte para muitos estudos, entretanto Chomsky propôs um falante-ouvinte ideal, em uma comunidade homogênea. Então, sentindo falta do caráter social e heterogêneo em uma mesma comunidade de fala, surge, em meados de 1960, um viés sociolinguístico, que considera o uso variável da língua. A Teoria da Variação e Mudança Linguística, também conhecida como Sociolinguística Quantitativa Laboviana (LABOV, 2008 [1972]) ou Sociolinguística Variacionista, formulada por Weinreich, Labov e Herzog (2006 [1968]), foi apresentada inicialmente em um simpósio em 1966, na Universidade do Texas. Mais tarde, o texto de Weinreich, Labov e Herzog foi publicado no livro Directions for historical linguistics, pela University of Texas Press, em 1968. Os autores afirmam que "os desvios de um sistema homogêneo não são todos eles erros aleatórios de desempenho, mas são num alto grau codificados e parte de uma descrição realista da competência de um membro de uma comunidade de fala" 
(WEINREICH; LABOV; HERZOG, 2006 [1968], p. 60). Desse modo, segundo Hora (2004, p. 19):

[...] a pesquisa sociolinguística implica levantamento cuidadoso dos registros de língua falada, descrevendo a variável (conjunto de variantes) e traçando um perfil das variantes (diversas maneiras de se dizer a mesma coisa em um mesmo contexto e com o mesmo valor de verdade ${ }^{1}$ ); análise dos fatores estruturais e sociais condicionantes; encaixamento da variável no sistema linguístico e social da comunidade; avaliação da variável, para a confirmação dos casos de variação ou mudança.

Tarallo e Kato (1989) apresentaram como a união das duas teorias, Teoria da Variação e Mudança linguística e Teoria de Princípios e Parâmetros, é efetiva para estudos sobre o português brasileiro.

\section{PRESSUPOSTOS METODOLÓGICOS}

Os dados foram recortados do Discurso semidirigido de inquéritos coletados pela equipe do Atlas Linguístico do Brasil (COMITÊ, 2001). O corpus é composto de 31 informantes (apesar de serem oito informantes por capital, quatro do sexo feminino e quatro do sexo masculino, uma informante de Cuiabá não precisou relatar por ter fornecido dados suficientes antes dessa parte), distribuídos por quatro capitais, Florianópolis, Curitiba, Cuiabá e Campo Grande, por dois graus de escolaridade, Ensino Fundamental e Ensino Universitário. O tratamento dos dados foi feito pelo Programa GoldVarb X (ROBINSON; LAWRENCE; TAGLIAMONTE, 2001) e os resultados são apresentados em percentual e em peso relativo se a variável for selecionada pelo programa como significativa.

\section{DESENVOLVIMENTO}

Após o tratamento dos dados realizado pelo Programa GoldVarb X (ROBINSON; LAWRENCE; TAGLIAMONTE, 2001), obteve-se um total de 1.406 ocorrências, sendo 1.157 casos de sujeito-pronome pleno (82,3\%), contra 249 de sujeito nulo $(17,7 \%)$.

Com relação à variável linguística pessoa gramatical (primeira, segunda e terceira pessoas), as ocorrências estão expostas na Tabela 1.

Tabela 1: Ocorrências de sujeitos plenos e nulos com relação à variável pessoa gramatical

\begin{tabular}{|c|c|c|}
\hline Pessoa Gramatical & $\begin{array}{c}\text { Pleno } \\
\text { (ocorrências/\%) }\end{array}$ & $\begin{array}{c}\text { Nulo } \\
\text { (ocorrências/\%) }\end{array}$ \\
\hline Primeira pessoa & $770(78,7 \%)$ & $208(21,3 \%)$ \\
\hline
\end{tabular}

\footnotetext{
${ }^{1}$ TARALLO, 2002 [1986], p. 8.
} 


\section{das Letras}

\begin{tabular}{|c|c|c|}
\hline Segunda pessoa & $62(98,4 \%)$ & $1(1,6 \%)$ \\
\hline Terceira Pessoa & $325(89 \%)$ & $40(11 \%)$ \\
\hline
\end{tabular}

Fonte: Elaborado pela autora com base nos resultados obtidos para a variável pessoa gramatical com dados do ALiB (COMITÊ, 2001).

Para a primeira pessoa, houve 770 ocorrências $(78,7 \%)$ de sujeitos plenos e 280 casos $(21,3 \%)$ de sujeitos nulos. De fato, o tipo de narrativa - relato - incita mais o uso da primeira pessoa, pois é solicitado que o informante relate algum acontecimento marcante em sua vida. Já para a segunda pessoa, 62 ocorrências de sujeitos plenos e 1 caso de nulo, hipotetiza-se que, por não haver tanta interação entre informante/entrevistador, é a pessoa gramatical com menos ocorrências. A terceira pessoa apresentou 325 ocorrências $(89 \%)$ de sujeitos plenos e 40 casos de sujeitos nulos $(11 \%)$; por vezes, os informantes não relatavam sobre a própria vida, mas sobre a vida de pessoas conhecidas ou sobre histórias que ouviram. Nas três pessoas gramaticais, encontra-se o predomínio do sujeito pleno; isso demonstra que o uso preenchido é o preferível em todas as pessoas gramaticais.

A Tabela 2 traz o número de ocorrências de sujeitos plenos e nulos por desinência verbal explícita ou implícita.

Tabela 2: Ocorrências de sujeitos plenos e nulos de acordo com desinência verbal explícita ou implícita

\begin{tabular}{|c|c|c|}
\hline Desinência verbal & $\begin{array}{c}\text { Pleno } \\
\text { (ocorrências/\%) }\end{array}$ & $\begin{array}{c}\text { Nulo } \\
\text { (ocorrências/\%) }\end{array}$ \\
\hline Explícita & $636(76,8 \%)$ & $192(23,2 \%)$ \\
\hline Implícita & $521(90,1 \%)$ & $57(9,9 \%)$ \\
\hline
\end{tabular}

Fonte: Elaborado pela autora com base nos resultados obtidos para a variável desinência verbal explícita/implícita com dados do ALiB (COMITÊ, 2001).

Com a desinência verbal explícita, houve 636 casos (76,8\%) de sujeitos plenos e 192 $(23,2 \%)$ de sujeitos nulos; em contrapartida, 521 ocorrências $(90,1 \%)$ de sujeitos plenos com desinência verbal implícita e $57(9,9 \%)$ de sujeitos nulos. Quando o verbo possui desinência verbal implícita, há mais preenchimento, no entanto também se verifica o predomínio do pleno mesmo em casos em que a desinência verbal é suficiente para reconhecer a pessoa gramatical.

Já a Tabela 3 mostra os casos de sujeitos plenos e nulos conforme a variável extralinguística sexo.

Tabela 3: Ocorrências de sujeitos plenos e nulos conforme a variável sexo 


\begin{tabular}{|c|c|c|}
\hline Sexo & $\begin{array}{c}\text { Pleno } \\
\text { (ocorrências/\%) }\end{array}$ & $\begin{array}{c}\text { Nulo } \\
\text { (ocorrências/\%) }\end{array}$ \\
\hline Masculino & $434(78,2 \%)$ & $121(21,8 \%)$ \\
\hline Feminino & $723(85 \%)$ & $128(15 \%)$ \\
\hline
\end{tabular}

Fonte: Elaborado pela autora com base nos resultados obtidos para a variável sexo com dados do ALiB (COMITÊ, 2001).

Averiguou-se que, para o sexo masculino, houve 434 casos $(78,2 \%)$ de sujeitos plenos e 121 ocorrências $(21,8 \%$ ) de sujeitos nulos; por sua vez, o sexo feminino produziu 723 casos (85\%) de sujeitos plenos e 128 (15\%) de sujeitos nulos. Labov (2008 [1972]) assevera que as mulheres são mais suscetíveis a usar a forma considerada inovadora e, embora haja um alto preenchimento por ambos os sexos, o sexo feminino fez mais uso da forma plena, "considerada a variante inovadora" (PAIVA; DUARTE, 2006, p. 136).

$\mathrm{Na}$ Tabela 4, apresentam-se as ocorrências de sujeitos plenos e nulos segundo a variável grau de escolaridade.

Tabela 4: Ocorrências de sujeitos plenos e nulos segundo a variável grau de escolaridade

\begin{tabular}{|c|c|c|}
\hline Grau de escolaridade & $\begin{array}{c}\text { Pleno } \\
\text { (ocorrências/\%) }\end{array}$ & $\begin{array}{c}\text { Nulo } \\
\text { (ocorrência/\%) }\end{array}$ \\
\hline Ensino Fundamental & $399(79,9 \%)$ & $106(21 \%)$ \\
\hline Ensino Médio & $758(84,1 \%)$ & $143(15,9 \%)$ \\
\hline
\end{tabular}

Fonte: Elaborado pela autora com base nos resultados obtidos para a variável grau de escolaridade com dados do ALiB (COMITÊ, 2001).

Com informantes que possuem Ensino Fundamental, verificou-se um total de 399 ocorrências $(79,9 \%)$ de sujeitos plenos e 106 casos $(21 \%)$ de sujeitos nulos. Para os portadores de Ensino Universitário, houve 758 ocorrências $(84,1 \%)$ de sujeitos plenos e 143 casos $(15,9 \%)$ de sujeitos nulos. O predomínio do sujeito pleno nos dois graus de escolaridade indica que mais anos de escolarização não impedem a forma preenchida do pronome; de fato, aqueles com Ensino Universitário preencheram mais do que os com Ensino Fundamental, o que permite dizer que talvez o fenômeno não seja rotulado como estigmatizado. localidade.

A Tabela 5 apresenta a distribuição de sujeitos plenos e nulos para a variável

Tabela 5: Ocorrências de sujeitos plenos e nulos para a variável localidade

\begin{tabular}{|c|c|c|}
\hline Localidades & $\begin{array}{c}\text { Pleno } \\
\text { (ocorrências/\%) }\end{array}$ & $\begin{array}{c}\text { Nulo } \\
\text { (ocorrências/\%) }\end{array}$ \\
\hline
\end{tabular}




\section{das Letras}

\begin{tabular}{|c|c|c|}
\hline Florianópolis & $415(88,7 \%)$ & $53(11,3 \%)$ \\
\hline Curitiba & $253(77,6 \%)$ & $73(22,4 \%)$ \\
\hline Cuiabá & $237(77,5 \%)$ & $69(22,5 \%)$ \\
\hline Campo Grande & $252(82,4 \%)$ & $54(17,6 \%)$ \\
\hline
\end{tabular}

Fonte: Elaborado pela autora com base nos resultados obtidos para a variável localidade com dados do ALiB (COMITÊ, 2001).

Com relação à variável localidade, houve mais ocorrências de sujeitos plenos na capital de Santa Catarina - Florianópolis - com 415 casos $(88,7 \%)$ de sujeitos plenos, contra $53(11,3 \%)$ de sujeitos nulos; em seguida, a capital do Mato Grosso do Sul, Campo Grande, apresentou 252 ocorrências (82,4\%) de sujeitos plenos e 54 (17,6\%) de sujeitos nulos; a seguir, a capital do Paraná - Curitiba - teve 253 ocorrências (77,6\%) de sujeitos plenos, contra 73 casos $(22,4 \%)$ de sujeitos nulos; na sequência, a capital do Mato Grosso, Cuiabá, com 237 ocorrências $(77,5 \%)$ de sujeitos plenos e, em contrapartida, 69 casos $(22,5 \%)$ de sujeitos nulos.

\section{CONSIDERAÇÕES FINAIS}

Como foi visto pelos resultados, o uso do sujeito pleno (82,3\%) é o preferido pelos 31 informantes. Ao analisar a similaridade entre as variáveis sexo, grau de escolaridade e localidade, verifica-se que esses fatores apresentam pequenas diferenças, mas o preenchimento predomina em todos, sendo um fenômeno geral e não um caso isolado. Os estudos com diversos corpora têm mostrado isso. Embora o português ainda não se comporte como línguas consideradas de sujeito preenchido, parece que caminha para essa direção.

\section{REFERÊNCIAS}

BOTASSINI, Jacqueline Ortelan Maia. A elipse do sujeito pronominal na linguagem falada do Paraná: uma análise variacionista, 1998, 105 folhas, Dissertação (mestrado em Linguística). Setor de Ciências Humanas, Letras e Artes, Universidade Federal do Paraná, Curitiba. CASTILHO, Ataliba Teixeira. Nova gramática do português brasileiro. São Paulo: Contexto, 2010.

COMITÊ NACIONAL DO PROJETO ALiB. Atlas linguístico do Brasil: questionários. Londrina: Eduel, 2001.

CHOMSKY, Noam. Aspects of the theory of syntax. Cambridge: MIT Press, 1965.

CHOMSKY, Noam. Lectures on government and binding. Dordrecht: Foris, 1981.

CHOMSKY, Noam. The minimalist program. Cambridge, MA: MIT Press, 1995.

DUARTE, Maria Eugênia Lamoglia. A perda do princípio "evite pronome" no português 
brasileiro, 1995, 151 folhas, Tese (doutorado Linguística). Instituto de Estudos da Linguagem, Universidade Estadual de Campinas, Campinas.

DUARTE, Maria Eugênia Lamoglia. Do pronome nulo ao pronome pleno: a trajetória do sujeito no português do Brasil. In: ROBERTS, Ian; KATO, Mary A. (Orgs.). Português Brasileiro: uma viagem diacrônica: homenagem a Fernando Tarallo. $2^{\mathrm{a}}$ ed. Campinas: Editora da Unicamp, 1996, p. 107-128.

DUARTE, Maria Eugênia Lamoglia. Avanço no estudo da mudança sintática associando a teoria da variação e mudança e a teoria de princípios e parâmetros. Caderno de Estudos Linguisticos, Campinas, v. 57, n. 1, p. 85-111, 2015.

DUARTE, Maria Eugênia Lamoglia. Empirismo e formalismo na investigação da mudança linguística em curso. Diadorim, v. 18, n. especial, p. 31-60, 2016.

DUARTE, Maria Eugênia Lamoglia. O sujeito nulo no Português Brasileiro. In: CASTILHO, Ataliba Teixeira; CYRINO, Sônia; MORAIS, Maria A. Torres. História do português brasileiro. Mudança sintática do português brasileiro: perspectiva gerativista, v. 6. São Paulo: Contexto, 2018, p. 28-71.

GALVES, Charlotte. O enfraquecimento da concordância no português brasileiro. In: ROBERTS, Ian; KATO, Mary A. (Orgs.). Português brasileiro: uma viagem diacrônica: homenagem a Fernando Tarallo. 2. ${ }^{a}$ ed. Campinas: Editora da Unicamp, 1996, p. 308-403. GALVES, Charlotte. Ensaios sobre as gramáticas do português. Campinas: Editora da Unicamp, 2001.

GRAVINA, Aline Peixoto. A natureza do sujeito nulo na diacronia do PB: estudo de um corpus mineiro (1845-1950), 2008, 146 folhas, Dissertação (mestrado em Linguística). Curso de Linguística do Instituto de Estudos da Linguagem, Universidade Estadual de Campinas, Campinas.

HORA, Dermeval. Teoria da variação: trajetória de uma proposta. In: HORA, Dermeval. (Org.). Estudos Sociolinguísticos: perfil de uma comunidade. João Pessoa: ILAPEC, 2004, p. 13-28.

HUANG, James C. T. On the distribution and reference of the empty categories. Linguistic Inquiry, 15, p. 531-574, 1984.

LABOV, William. Padrões sociolinguísticos. Tradução de Marcos Bagno, Maria Marta Pereira Scherre, Caroline Rodrigues Cardoso. São Paulo: Parábola Editorial, 2008.

MAGALHÃES, Telma Moreira Vianna. O sistema pronominal sujeito e objeto na aquisição do português europeu e do português brasileiro, 2006, 187 folhas, Tese (doutorado em Linguística). Instituto de Estudos da Linguagem, Universidade Estadual de Campinas, Campinas.

MARINS, Juliana Esposito. O parâmetro do sujeito nulo: uma análise contrastiva entre o português e o italiano, 2009, 111 folhas, Dissertação (mestrado em Letras Vernáculas). Faculdade de Letras, Universidade Federal do Rio de Janeiro, Rio de Janeiro.

MENON, Odete Pereira da Silva. O sistema pronominal do português do Brasil. Revista Letras, v. 44, p. 91-106, 1995.

MENON, Odete Pereira da Silva. Uso do pronome sujeito de primeira pessoa no português do Brasil. Organon (UFRGS), Porto Alegre, v. 14, n. 28-29, p. 157-177, 2002.

MIOTO, Carlos; SILVA, Maria Cristina Figueiredo; LOPES, Ruth. Novo manual de sintaxe. São Paulo: Contexto, 2016. 
MONTEIRO, José Lemos. Pronomes pessoais. Subsídios para uma gramática do português do Brasil. Fortaleza: EUFC, 1994.

PAIVA, Maria da Conceição; DUARTE, Maria Eugênia Lamoglia. Quarenta anos depois: a herança de um programa na sociolinguística brasileira. In: WEINREICH, Uriel; LABOV, William; HERZOG, Marvin. Fundamentos empiricos para uma teoria da mudança linguística. Tradução de Marcos Bagno; revisão técnica de Carlos Alberto Faraco; posfácio de Maria da Conceição A. de Paiva, Maria Eugênia Lamoglia Duarte. São Paulo: Parábola Editorial, p. 131-151, 2006 [1968].

PERINI, Mário Alberto. A gramática gerativa: introdução ao estudo da sintaxe portuguesa. Belo Horizonte: Vigília, 1979.

ROBINSON, John; LAWRENCE, Helen; TAGLIAMONTE; Sali. GoldVarb 2001: a multivariate analysis. User's manual. Outubro, 2001.

SILVA, Humberto Soares da. O parâmetro do sujeito nulo: confronto entre o português e o espanhol, 2006, 119 folhas, Dissertação (mestrado em Letras Vernáculas). Faculdade de Letras, Universidade Federal do Rio de Janeiro, Rio de Janeiro.

SILVA, Humberto Soares da. Evidências da mudança em dados da língua -E: o sujeito pronominal no português e no espanhol, 2011, 143 folhas, Tese (doutorado em Letras Vernáculas). Faculdade de Letras, Universidade Federal do Rio de Janeiro, Rio de Janeiro. TARALLO, Fernando; KATO, Mary. Harmonia trans-sistêmica: variação intra- e interlinguística. Preedição 5, Campinas: Editora da Unicamp, 1989.

WEINREICH, Uriel; LABOV, William; HERZOG, Marvin. Fundamentos empiricos para uma teoria da mudança linguística. São Paulo: Parábola, 2006 [1968].

Recebido em: 28/02/2019

Aprovado em: 07/06/2019

Publicado em: 28/09/2019 